\title{
Strong quantum interference in strongly disordered bosonic insulators
}

\author{
S.V. Syzranov ${ }^{1,2}$, A. Moor $^{1}$, K.B. Efetov ${ }^{1}$ \\ ${ }^{1}$ Theoretische Physik III, Ruhr-Universität Bochum, 44780 Bochum, Germany, \\ ${ }^{2}$ Institute for Theoretical Condensed Matter Physics, \\ Karlsruhe Institute of Technology, 76131 Karlsruhe, Germany
}

(Dated: February 21, 2018)

\begin{abstract}
We study the variable-range hopping (VRH) of bosons in an array of sites with short-range interactions and a large characteristic coordination number. The latter leads to strong quantum interference phenomena yet allows for their analytical study. We develop a functional renormalization group scheme that repeatedly eliminates high-energy sites properly renormalizing the tunnelling between the low-energy ones. Using this approach we determine the temperature and magnetic field dependence of the hopping conductivity and find a large positive magnetoresistance. With increasing magnetic field the behaviour of the conductivity crossovers from the Mott's law to an activational behaviour with the activation gap proportional to the magnetic field.
\end{abstract}

PACS numbers: 72.20.-i, 72.20.Ee, 73.23.Hk, 05.60.Gg

Transport in a huge variety of insulators is described by the model of variable-range hopping (VRH) [1] based on a few rather general assumptions: charge carriers hop inelastically between random-energy sites, e.g. impurities, grains, or (quasi-)localized states, the probability of a hop exponentially decreases with distance and has an activational temperature dependence. The particular nature of the sites and the bath is not important,the model can be applied to the transport of electrons in doped semiconductors as well as to that of Cooper pairs in macroscopically insulating superconductive materials.

In the course of each inelastic hop between two sites a charge carrier may tunnel elastically via intermediate sites. The interference between different-path contributions gives rise to mesoscopic and magneto-transport phenomena 2]. The interference is suppressed in strongly insulating materials because there the elastic transmission is dominated either by the direct tunnelling path between two sites or by the shortest chain of intermediary sites. With increasing the intersite couplings or the site concentration, more alternative paths come into play and the interference effects get more pronounced.

This Letter is devoted to the study of the VRH transport in bosonic insulators with large coordination numbers. In these materials the interference phenomena are particularly strong because of the constructive interference between nearly all tunnelling processes, including the many-body ones. Large coordination number $Z \gg 1$ of the effective array of sites allows us to treat the interference effects analytically, unlike the previous VRH studies, and is characteristic of a broad class of insulating materials. Those include, for example, superconductive films close to the metal-insulator transition (see, e.g., Ref. [3] for a review), densely packed granular materials, disordered bosonic systems with many particles on the localization length, "superconductive hay" [4], etc. Strong interference in these materials manifests itself, for instance, in a huge magnetoresistance [5],-- magnetic field may change conductance by orders of magnitude or even drive a superconductor into a strong Cooper-pair insulator. Transport phenomena and superconductor-insulator transitions are also being observed since recently in artificially made arrays of Josephson junctions with $Z \gg 1[\underline{6}$.

In general, the interference in these materials is intractable perturbatively, unlike many earlier VRH models (cf., e.g., Ref. 7]). Tunnelling along paths of sites with close energies [8] is also irrelevant for such systems due to the small portion of such paths among all possible ones.

Model. Although we focus on the transport of bosons, the results of this Letter apply also to systems with an arbitrary charge carrier statistics if the VRH is dominated by single-particle hops. The latter may be the case, for instance, in an electronic system with a shallow Fermi sea, that is sufficiently exceeded by the characteristic Coulomb energy. As we consider systems with $Z \gg 1$, we also expect the results to hold qualitatively in the materials with coordination numbers of order unity.

We assume for simplicity that the intersite interactions are short-range and the number of particles on each site uniquely determines their state. This simplification is possible if the other states are absent or separated by large energy gaps, which is characteristic of electrons in doped semiconductors 9], Cooper-pairs in macroscopically insulating media [10, 11], and of the charge carriers in nearly all other materials under consideration.

The particle motion is governed by the Hamiltonian

$$
\hat{\mathcal{H}}=\sum_{\mathbf{r}} U_{\mathbf{r}}\left(n_{\mathbf{r}}\right)-\sum_{\mathbf{r} \neq \mathbf{r}^{\prime}} t_{\mathbf{r} \mathbf{r}^{\prime}} \hat{b}_{\mathbf{r}}^{\dagger} \hat{b}_{\mathbf{r}^{\prime}}
$$

Here $U_{\mathbf{r}}\left(n_{\mathbf{r}}\right)$ is the energy of $n_{\mathbf{r}}$ particles on site $\mathbf{r}$, a large random quantity affected by disorder and stray charges close to the site. The last term in Eq. (1) accounts for the intersite hopping, $\hat{b}_{\mathbf{r}}^{\dagger}$ and $\hat{b}_{\mathbf{r}}$ being (bosonic) creation and annihilation operators on grain $\mathbf{r}, n_{\mathbf{r}}=\hat{b}_{\mathbf{r}}^{\dagger} \hat{b}_{\mathbf{r}}$.

Below we count the energies of all excitations from the 
energy of the "ground charging state", the configuration of integers $n_{\mathbf{r}}$ that minimize the first term in the righthand side (rhs) of Eq. (1). Typically, the energies $E_{\mathrm{r}}^{+}$and $E_{\mathbf{r}}^{-}$, needed respectively to add or remove a particle from the site $\mathbf{r}$, are determined by the Coulomb interactions with the other particles and stray charges, and we refer to these energies and on-site excitation states as respectively "charging" energies and states.

As only low-energy states are relevant for lowtemperature conductance, one can conveniently rewrite the Hamiltonian in a reduced Hilbert space,-- each site $\mathbf{r}$ has only 3 states: $| \pm 1\rangle_{\mathbf{r}}$ and $|0\rangle_{\mathbf{r}}$, corresponding respectively to \pm 1 and 0 extra particles as compared to the ground charging state. Introducing spin-1 operators; $S_{\mathbf{r}}^{z}| \pm 1\rangle_{\mathbf{r}}= \pm| \pm 1\rangle_{\mathbf{r}}, S_{\mathbf{r}}^{z}|0\rangle=0, S_{\mathbf{r}}^{ \pm}|\mp 1\rangle=\sqrt{2}|0\rangle$, $S_{\mathbf{r}}^{ \pm}|0\rangle=\sqrt{2}| \pm 1\rangle$, we arrive at the reduced Hamiltonian

$$
\begin{aligned}
\hat{\mathcal{H}}= & \frac{1}{2} \sum_{\mathbf{r}}\left[\left(E_{\mathbf{r}}^{+}+E_{\mathbf{r}}^{-}\right) \hat{S}_{\mathbf{r}}^{z} \hat{S}_{\mathbf{r}}^{z}+\left(E_{\mathbf{r}}^{+}-E_{\mathbf{r}}^{-}\right) \hat{S}_{\mathbf{r}}^{z}\right] \\
& -\frac{1}{2} \sum_{\mathbf{r}, \mathbf{r}^{\prime}} J_{\mathbf{r r}^{\prime}} \hat{S}_{\mathbf{r}}^{+} \hat{S}_{\mathbf{r}^{\prime}}^{-},
\end{aligned}
$$

where the exact form of the coupling constants $J_{\mathbf{r r}^{\prime}}$ depends on the microscopic details of the model. For instance, for a bosonic system with large occupancies $n_{\mathbf{r}} \gg$ 1 in the charging ground state, $J_{\mathbf{r r}^{\prime}}=\left(n_{\mathbf{r}} n_{\mathbf{r}^{\prime}}\right)^{1 / 2} t_{\mathbf{r r}^{\prime}}$.

In absence of inter-site interactions at strong disorder, the density of states (DoS) of the (anti)particle states has no Coulomb gap 9] and, thus, reaches some finite value $\nu$ at low energies. The assumption of a constant DoS in a certain energy interval is crucial for our consideration.

Site-decimating renormalization group. The transport of low-energy excitations can be analysed by constructing a logarithmic renormalization group (RG) technique that repeatedly eliminates the highest-energy charging states properly renormalizing the intersite tunnelling couplings between all the lower-energy states.

Let us select a few (anti-)particle charging states with the very highest energies and split the Hamiltonian into the Hamiltonian $\hat{\mathcal{H}}_{\text {high }}$ of the respective high-energy charging states, the Hamiltonian $\hat{\mathcal{H}}_{\text {low }}$ of the rest of the system, and the tunnelling $\hat{V}$ between the selected states and all the others. Any eigenstate of the system is, strictly speaking, a superposition of the charging states on all sites. The projection $\Psi_{\text {low }}$ of the eigenfunction on the lower-energy charging states satisfies Schrödinger equation $\hat{\mathcal{H}}_{\text {low }}^{\prime} \Psi_{\text {low }}=\varepsilon \Psi_{\text {low }}$ with an effective (exact) Hamiltonian

$$
\hat{\mathcal{H}}_{\text {low }}^{\prime}=\hat{\mathcal{H}}_{\text {low }}-\hat{V}\left(\hat{\mathcal{H}}_{\text {high }}-\varepsilon\right)^{-1} \hat{V},
$$

$\varepsilon$ being the eigenenergy. The latter may be neglected in Eq. (3) if the energies of the selected states significantly exceed the energies of the other states.

Assume, the intersite tunnelling elements are small, so that one can neglect (anti-)particle hopping between the highest-energy charging states. Then we find that the modified Hamiltonian (3) has the same form as the initial Hamiltonian (2) provided the couplings are renormalized,

$$
J_{\mathbf{r r}^{\prime}} \rightarrow J_{\mathbf{r r}^{\prime}}+\sum_{\mathbf{R}_{p}} \frac{J_{\mathbf{r} \mathbf{R}_{p}} J_{\mathbf{R}_{p} \mathbf{r}^{\prime}}}{E_{\mathbf{R}_{p}}^{+}}+\sum_{\mathbf{R}_{h}} \frac{J_{\mathbf{r R}_{h}} J_{\mathbf{R}_{h} \mathbf{r}^{\prime}}}{E_{\mathbf{R}_{h}}^{-}}
$$

where $\mathbf{R}_{p}$ and $\mathbf{R}_{h}$ label the eliminated particle and antiparticle states respectively. The increment of the magnitude of the coupling $\left|J_{\mathbf{r r}^{\prime}}\right|$ is positive, as the energies $E_{\mathbf{R}_{p}}^{+}$and $E_{\mathbf{R}_{h}}^{-}$in Eq. (4) are positive by definition.

Eq. (4) can be understood as follows. In addition to the direct tunnelling between sites $\mathbf{r}$ and $\mathbf{r}^{\prime}$ a particle may cotunnel via virtual high-energy states in one of two ways: 1)via an intermediate particle state on site $\mathbf{R}$, or 2)first create a particle-antiparticle dipole on sites $\mathbf{r}^{\prime}, \mathbf{R}$, then-on sites $\mathbf{R}, \mathbf{r}$. The respective high-energy particle or antiparticle state on the site $\mathbf{R}$ can be omitted provided the coupling constants $J_{\mathbf{r r}^{\prime}}$ are renormalized. Since processes 1) and 2) interfere constructively in a bosonic system, the renormalization increases the tunnelling.

The generic Eq. (4) may be of little use for arbitrary distributions of the site energies and locations. However, in the case of a large coordination number $Z$ the averaging over the site positions leads, as we show below, to a simple expression involving the averaged function $J_{\mathbf{r r}^{\prime}}$. Moreover, large coordination number suppresses the fluctuations of strongly renormalized couplings, since all the decimated states contribute with the same sign to each coupling, while the fluctuations result from multiple random-sign contributions.

Repeatedly eliminating the highest-energy charging (anti-)particle states and introducing continuous coordinates in the coupling function we arrive at a functional RG equation with a running high-energy cutoff $E$ :

$$
\partial_{l} J_{\mathbf{r r}^{\prime}}=\lambda \int J_{\mathbf{r R}} J_{\mathbf{R r}^{\prime}} d \mathbf{R}, \quad l=\log \left(E_{0} / E\right),
$$

where the quantity $\lambda=2 \nu$ is independent of energy, as we assumed previously.

On each RG step we considered the cotunnelling via only one intermediate site, which is justified for sufficiently small couplings $J$,

$$
\lambda J \equiv \int \lambda J_{\mathbf{r r}} d \mathbf{r}^{\prime} \ll 1 .
$$

Indeed, taking into account the tunnelling between eliminated charging states in the $n$-th order results in $\sim$ $\left[(\lambda J) \log \left(E^{\prime} / E\right)\right]^{n}$ relative correction to the tunnelling couplings on an RG step with energy rungs $E^{\prime}$ and $E$. Thus, Eq. (5) is the one-loop functional RG equation for the intersite tunnelling elements.

Eq. (5) should be solved implying the coupling $J_{\mathbf{r r}^{\prime}}$ corresponds to the bare one at the energies of order of the characteristic charging energy $E_{0}$ in the nonrenormalized Hamiltonian (2). The RG flow must be 
stopped if, as a result of the renormalization, the couplings become too large, violating condition (6), or if the high-energy cutoff $E$ reaches either some characteristic energy of relevant conducting excitations or the mean level spacing $1 /\left(\nu \xi^{d}\right)$ on the characteristic radius $\xi$ of the renormalized function $J_{\mathbf{r r}^{\prime}}$ in a $d$-dimensional space.

The smallness of the fluctuations of the coupling on each RG step requires $Z\left(E / E^{\prime}\right)\left[\ln \left(E^{\prime} / E\right)\right]^{2} \gg 1$. So long as the fluctuations are suppressed, the renormalized function $J_{\mathbf{r r}^{\prime}}$ fully describes the conduction. We emphasize that this is characteristic of a bosonic system, considered in the present Letter, and makes our analysis very different from that of fermionic insulators 2, 12]. In a fermionic system our RG scheme would yield a non-renormalized coupling function with large fluctuations, which would determine then the transport properties of the system. The latter does not apply, however, to a system with a so shallow Fermi sea that the transport is effectively singleparticle,- the renormalization then occurs similarly to the bosonic case.

Mean-field critical point. In absence of magnetic field the intersite tunnelling is translationally invariant, $J_{\mathbf{r r}^{\prime}}=$ $J_{\mathbf{r}-\mathbf{r}^{\prime}}$. Then for the Fourier-transorm $J_{\mathbf{k}}$ of the coupling function Eq. (5) yields

$$
J_{\mathbf{k}}(l)=J_{\mathbf{k}}(0)\left[1-\lambda J_{\mathbf{k}}(0) l\right]^{-1} .
$$

where $J_{\mathbf{k}}(0)$ is the bare value of the tunnelling amplitude. In the limit of a strong insulator, $\lambda J_{\mathbf{k}} l \ll 1$, the renormalization results in a small correction to the tunnelling and can also be obtained perturbatively by considering the tunnelling processes via one intermediate site.

The renormalization is more pronounced in less insulating materials. In particular, the renormalized tunnelling elements diverge if the coordination number $Z$ or the bare coupling are sufficiently large, namely, if the quantity $J_{\mathbf{k}}(0)$ exceeds some critical value $J_{c}$,

$$
\lambda J_{c} l_{\max }=1,
$$

$l_{\max }$ being the maximal value of the logarithm that stops the RG flow.

Eq. (8) matches the mean-field (MF) criterion of the phase transition in a system with Hamiltonian (2) and the uniform order parameter $\Delta=\left\langle\hat{S}_{\mathbf{r}}^{+}\right\rangle$. However, we emphasize that the above analysis is not sufficient to prove the existence of the metal-insulator or superconductorinsulator transition when condition (8) is fulfilled, because we have to stop the RG flow if the inequality (6) no longer applies. Clearly, to study such transitions one has to employ alternative methods, which is beyond the scope of this paper. Now, we concentrate on the transport in the insulating regime when the effective amplitude $J_{\mathbf{k}}(l)$, Eq. (7), is still small.

Conductivity away from the MF transition point. We can find rather easily the temperature dependency of conductivity in the region

$$
l_{\max }^{-1} \ll 1-J(0) / J_{c} \ll 1 .
$$

Eq. (9) means that the system is quite close to the MF transition and the effective tunnelling amplitude $J_{\mathbf{k}}(l)$ is strongly renormalized, although being small. Deeper in the insulating regime, i.e. at smaller $J(0)$ than that in Eq. (9), the renormalization is negligible and so is the effect of the cotunnelling on the VRH.

Defining the characteristic radius $\zeta$ of the nonrenormalized intersite coupling as

$$
J_{\mathbf{k}}(0) \approx J(0)\left(1-\zeta^{2} \mathbf{k}^{2}\right), \quad \mathbf{k} \rightarrow 0
$$

we find from Eq. (77) the dependence of the tunnelling on distance $\left|\mathbf{r}-\mathbf{r}^{\prime}\right| \gg \zeta$ :

$$
\begin{array}{r}
J_{\mathbf{r r}^{\prime}}(l)=J(0) \frac{\xi^{2}}{\zeta^{2}} \int \frac{d \mathbf{k}}{(2 \pi)^{d}} \frac{e^{i \mathbf{k}\left(\mathbf{r}-\mathbf{r}^{\prime}\right)}}{1+\xi^{2} k^{2}} \\
\sim J(0) \xi^{2-d} \zeta^{-2}\left|\mathbf{r}-\mathbf{r}^{\prime}\right|^{2-d} \exp \left(-\left|\mathbf{r}-\mathbf{r}^{\prime}\right| / \xi\right),
\end{array}
$$

where we defined the characteristic radius

$$
\xi=\zeta\left[(\lambda J(0) l)^{-1}-1\right]^{-1 / 2}
$$

of the renormalized coupling function.

At sufficiently low temperatures the RG flow should be stopped if the characteristic number $Z(l)=E \nu \xi^{d}$ of states on length $\xi$ approaches unity. Eq. (11) and $l=$ $\ln \left(E_{0} / E\right)$ define self-consistently the energy $E=\left(\nu \xi^{d}\right)^{-1}$ that terminates the flow.

Thus, applying the RG procedure we arrive at a sparse array of sites with the coordination number $Z$ of order unity, the characteristic intersite spacing $\xi$, Eq. (11), characteristic on-site energies $E_{\min }=1 /\left(\nu \xi^{d}\right)$, and the tunnelling amplitudes given by Eq. (10). Inelastic hopping in the latter model is dominated by the direct hops between the sites, while the interference only slightly modifies the behaviour of the particle wavefunctions.

Owing to the weakness of the tunnelling, each particle excitation is almost entirely localized on one site. By construction of the site-decimating RG the particle amplitude distance $r$ away from this site estimates

$$
\Psi(r) \sim J_{0 \mathbf{r}}\left[\ln \left(E_{0} / E_{r}\right)\right] / E_{r}, E_{r}=\max \left[\left(\nu r^{d}\right)^{-1}, E_{\min }\right]
$$

in the first order in the small tunnelling. At large distances the wavefunction decays exponentially $\propto$ $\exp (-r / \xi)$, cf. Eqs. (10) and (12). Applying the traditional Mott's arguments [1] we find the temperature dependence of conductivity (Mott's law):

$$
\sigma=\sigma_{0} \exp \left[-\left(T_{M}^{\xi} / T\right)^{1 /(d+1)}\right], T \ll T_{M}^{\xi} \sim\left(\nu \xi^{d}\right)^{-1},
$$

with a renormalized Mott's temperature $T_{M}^{\xi}$ matching the value of the cutoff $E_{\min }$ in the RG procedure.

At $T>T_{M}^{\xi}$ the RG should be stopped when the running energy cutoff reaches temperature $T$, as the latter sets the characteristic energy of conducting excitations. The power-lower hopping probability on distances 
shorter than $\xi$ leads to the temperature dependency of conductivity weaker than exponential. The standard Mott's arguments yield[13] a power law $\sigma \sim T^{\alpha}$, although those cannot be used to find explicitly the power $\alpha$.

Effect of magnetic field. In magnetic field each tunnelling amplitude acquires a phase:

$$
J_{\mathbf{r}_{1} \mathbf{r}_{2}}=\left|J_{\mathbf{r}_{1} \mathbf{r}_{2}}\right| \exp \left[i q c^{-1} \int_{\mathbf{r}_{1}}^{\mathbf{r}_{2}} \mathbf{A}(\mathbf{r}) d \mathbf{r}\right] .
$$

Here $q$ is the particle charge and the integration is carried out along a certain path connecting sites $\mathbf{r}_{1}$ and $\mathbf{r}_{2}$, which in general depends on the microscopic details and for simplicity assumed below to be a straight line. We neglect the modification of the non-renormalized values of the couplings $\left|J_{\mathbf{r}_{1} \mathbf{r}_{2}}\right|$ due to the "shrinkage of wavefunctions" [9]. In principle, it can be straightforwardly included in the magnetoconductance.

The RG procedure leaves the phase factors intact but modifies the tunnelling amplitudes as

$$
\partial_{l}\left|J_{\mathbf{r r}^{\prime}}\right|=\lambda \int\left|J_{\mathbf{r R}}\right|\left|J_{\mathbf{R r}^{\prime}}\right| \exp \left[i q c^{-1} \oint_{\mathbf{r R r}^{\prime}} \mathbf{A}(\mathbf{r}) d \mathbf{r}\right] d \mathbf{R} \text {. }
$$

The contour integral in Eq. (15) gives the magnetic flux through the triangular contour $\mathbf{r} \rightarrow \mathbf{R} \rightarrow \mathbf{r}^{\prime} \rightarrow \mathbf{r}$.

Let us consider a two-dimensional system in a weak magnetic field $B$, so that the renormalization is not suppressed and the length

$$
L_{B}=[c /(q B)]^{1 / 2}
$$

exceeds the characteristic coupling radius $\xi$ in the absence of magnetic field, Eq. (11). Then the RG should be stopped again at the same high-energy cutoff $\left(\nu \xi^{d}\right)^{-1}$.

According to Eq. (15), the tunnelling on distance $r$ is unaffected by magnetic field if the flux through the characteristic "interference area" 7] $r^{3 / 2} \xi^{1 / 2}$ is less than $c / q$, i.e. if $r^{3 / 2} \xi^{1 / 2}<L_{B}^{2}$. Therefore, the magnetic field has no effect on conductance provided the mean inelastic hopping distance[1] $\bar{r} \sim \xi\left(T_{M}^{\xi} / T\right)^{1 / 3}$ does not exceed the scale $R \sim L_{B}^{4 / 3} \xi^{-1 / 3}$, which otherwise determines the characteristic length of the inelastic hopping, as it serves as a cutoff of the tunnelling $J_{\mathbf{r r}^{\prime}}$ and the excitation wavefunctions, Eq. (12).

At larger magnetic fields, $\xi>L_{B}$, the RG flow should be stopped when the number of remaining states on length $L_{B}$ approaches unity, and the renormalized coupling is cut on the length $L_{B}$, Fig. 1. The conduction comes then from the inelastic hopping on distance $R \sim$ $L_{B}$ between sites of the characteristic energy $1 /\left(\nu L_{B}^{2}\right)$.

Thus, we arrive at the conductivity

$$
\sigma=\sigma_{0} \exp \left[-\frac{1}{\nu T} \frac{\min \left(\xi^{2 / 3}, L_{B}^{2 / 3}\right)}{L_{B}^{8 / 3}}\right]
$$

that shows an activational behaviour with the activation gap $\propto B$ in high magnetic fields and $\propto B^{4 / 3}$ in lower

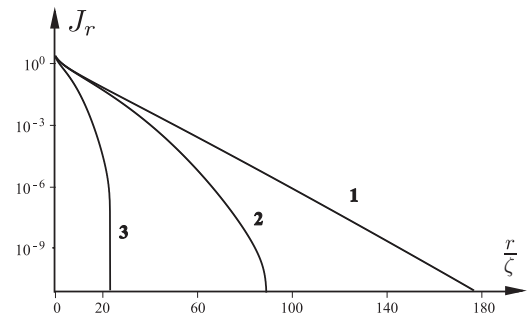

FIG. 1. The dependence of the renormalized tunnelling on distance, obtained from the numerical solution of Eq. (15). Three curves correspond to different values of magnetic field [in units $\left.\left.\left.\left.c /\left(q \zeta^{2}\right)\right] \mathrm{B}=1\right) 0,2\right) 0.001,3\right) 0.01 . l=0.97 l_{\max }$.

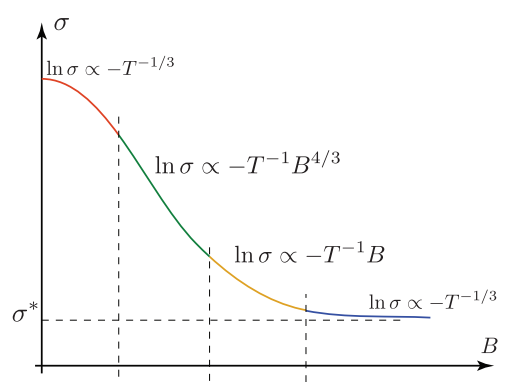

FIG. 2. (Colour online) The dependence of conductivity on magnetic field in a two-dimensional system.

fields, Fig. 2. Upon decreasing the field so that $T / T_{M}^{\xi} \gtrsim$ $\left(\xi / L_{B}\right)^{4}$ the conductivity (17) crossovers to Eq. (13).

At very strong magnetic fields, $B \ggg c /\left(q \zeta^{2}\right)$, the interference between the tunnelling paths is destroyed, which suppresses the renormalization and leads to the usual Mott's-law conductivity, Eq. (13), with $\xi=\zeta$.

Recently a similar model of Cooper-pair hopping in an array of sites with random energies and large coordination number $Z \gg 1$ has been studied in Refs. [3, 11] in order to describe the superconductor-insulator transition (SIT) in disordered superconductive films. In Ref. 11] the effective array of sites has been mapped on a Bethe lattice and studied by means of an MF-type approach with a site-dependent order parameter, using $Z \gg 1$. We disagree with such a mapping because Bethe lattice contains no finite loops of sites and, thus, cannot account properly for the interference phenomena considered here. Actually, the mapping of Ref. [11] cannot be justified even after performing the renormalization, because the RG flow can be stopped only when the characteristic coordination number $Z(l)$ reaches unity.

An interesting issue that deserves a separate investigation is the role of the intersite interactions in the SIT in disordered superconductive films, as even arbitrarily small long-range charging interactions are known [14 to renormalize the parameters of a clean system and change the order of the phase transition.

Conclusion. We considered low-temperature variablerange hopping transport of bosons in an array of sites 
with random energies. We constructed a renormalization group technique that allows one to repeatedly eliminate the highest-energy on-site states, renormalizing the intersite couplings between the lower-energy ones. Using this procedure, we reduced the system to a sparse array of sites and found the dependency of conductivity on temperature and magnetic field, Eqs. (13) and (17). When increasing magnetic field the dependency crossovers from the Mott's law to Arrhenius law with the activation temperature proportional to the magnetic field.

Acknowledgements. Our work has been supported by SFB Transregio 12 and SFB 491. We have benefited from discussions with L.B. Ioffe, V.E. Kravtsov, M. Müller, B.I. Shklovskii, and B. Spivak.

[1] N. F. Mott and E. A. Davis, Electronic processes in noncrystalline materials (Clarendon, Oxford, 1979).

[2] B. I. Shklovskii and B. Z. Spivak in Hopping Transport in Solids, eds. M. Pollak, B. Shklovskii, North-Holland, Amsterdam (1990).

[3] M. V. Feigelman, L. B. Ioffe, V. E. Kravtsov, and E. Cuevas, Annals of Physics 325, 1390 (2010).

[4] V. M. Vinokur, L. B. Ioffe, A. I. Larkin, and M. V. Feigelman, Sov. Phys. JETP 66, 198 (1987).

[5] G. Sambandamurthy, L. W. Engel, A. Johansson, and
D. Shahar, Phys. Rev. Lett. 92, 107005 (2004); T. I. Baturina, C. Strunk, M. R. Baklanov, and A. Satta, Phys. Rev. Lett. 98, 127003 (2007); H. S. J. van der Zant, W. J. Elion, L. J. Geerligs, and J. E. Mooij, Phys. Rev. B 54, 10081 (1996); Y.-H. Lin and A. M. Goldman, Phys. Rev. Lett. 106, 127003 (2011); V. F. Gantmakher and V. T. Dolgopolov, Usp. Fiz. Nauk 53, 1 (2010).

[6] J. Paramanandam, M. T. Bell, L. B. Ioffe, and M. E. Gershenson, arXiv:1112.6377.

[7] M. E. Raikh and G. F. Wessels, Phys. Rev. B 47, 15609 (1993); W. Schirmacher, Phys. Rev. B 41, 2461 (1990).

[8] U. Sivan, O. Entin-Wohlman, and Y. Imry, Phys. Rev. Lett. 60, 1566 (1988); Y. Meir, N. S. Wingreen, O. EntinWohlman, and B. L. Altshuler, Phys. Rev. Lett. 66, 1517 (1991).

[9] B. I. Shklovskii and A. L.Efros, Electronic properties of doped semiconductors (Springer, Heidelberg, 1984).

[10] K. B. Efetov, Sov. Phys. JETP 51, 1015 (1980).

[11] L. Ioffe and M. Mezard, Phys. Rev. Lett. 105, 037001 (2010); M. V. Feigelman, L. B. Ioffe, and M. Mezard, Phys. Rev. B 82, 184534 (2010).

[12] H. L. Zhao, B. Z. Spivak, M. P. Gelfand and S. Feng, Phys. Rev. B 44, 10760 (1991)

[13] Y. K. Vekilov and E. I. Isaev, Phys. Lett. A 338, 66 (2005).

[14] M. P. A. Fisher and G. Grinstein, Phys. Rev. Lett. 60, 208 (1988); S. V. Syzranov, I. L. Aleiner, B. L. Altshuler, and K. B. Efetov, Phys. Rev. Lett. 105, 137001 (2010). 